\title{
Valuation of Cash Flows under Random Rates of Interest: A Linear Algebraic Approach
}

\author{
P. Date ${ }^{\mathrm{a}, *}$, R. Mamon ${ }^{\mathrm{a}}$, I.C. Wang ${ }^{\mathrm{a}}$ \\ ${ }^{a}$ Center for the Analysis of Risk and Optimisation Modelling Applications, School \\ of Information Systems, Computing and Mathematics, Brunel University, \\ Middlesex, UB8 3PH UK. Phone: +44 1895 274000. Fax: +44 1895269732.
}

\begin{abstract}
This paper reformulates the classical problem of cash flow valuation under stochastic discount factors into a system of linear equations with random perturbations. Using convergence results, a sequence of uniform approximations is developed. The new formulation leads to a general framework for deriving approximate statistics of cash flows for a broad class of models of stochastic interest rate process. We then show applications of the proposed method by pricing default-free and defaultable bonds. The methodology developed in this paper is applicable for a variety of uncertain cash flow analysis problems.
\end{abstract}

Key words: Statistical methods, stochastic interest rate models

\section{Introduction}

The problem of calculating the present value of future cash flows is fundamental in contingent claim analysis and in investment appraisal problems. Financial instruments such as pensions, insurance, bonds, mortgages and more recently derivatives contracts need to be valued incorporating interest rate risk. Many of these securities consist of a series of periodic payments called annuities. The classic literature on pricing annuities concentrates on deterministic discount factor as described in Kellison (1991). Bowers et al. (1997) introduced the valuation of annuities when interest rates are random variables. However, there is no explicit reference to risk-neutral pricing and no real dynamics for the interest rate is suggested. Random interest rate formulation is further explored in Zaks (2001) whose characterisation

\footnotetext{
* Corresponding author.

Email addresses: paresh.date@brunel.ac.uk (P. Date), rogemar.mamon@brunel.ac.uk (R. Mamon), mapgicw@brunel.ac.uk (I.C. Wang).
} 
focused on the mean and variance of the accumulation factor assuming rates are independent, identically distributed random variables. Certain results from Zak's formulations were later modified by Burnecki, Marciniuk and Weron (2003).

In this paper, we consider stochastic interest rates and work in a valuation formula wherein the present value of a claim is the average of its discounted value over all possible positive future interest rate paths in a risk-neutral framework. Here, the discount factor is given by the cumulative one-period rates along the future path. We assume the existence of riskneutral specification as explained in Cheyette (1998). Hamilton (1988) also employed this framework to test the unbiased expectation hypothesis of the term structure of interest rates.

Unlike common approaches in term structure modelling such as Monte-Carlo methods (Cheyette (1998) for example), lattice technique (Jarrow (2002) for example) and direct calculation of expectation under a martingale measure, we reduce the valuation problem into a problem of inverting a matrix with random perturbations. Formulae for accurate approximation of bond prices are derived in terms of joint conditional moments of one period, future spot rates. We show how to obtain the joint moments of future spot rates in terms of the parameters of standard short rate models.

The issue of positive interest rates is also addressed as we can begin with positive rates and control the perturbation. Consequently, this offers an alternative perspective to the studies conducted by Koch and Schepper (2004) and Schepper et al. (1997) attempting to restrict interest rate evolution in order to meet special types of financial or actuarial constraints.

The scheme of this paper is as follows. The next section defines notation and sets up the matrix inversion problem mentioned above. The succeeding three sections discuss approximate solution and demonstrate certain applications. The final section summarises the contributions and provides directions for future research.

\section{The Cash flow Valuation Problem and the Linear System of Equations}

Throughout this paper, boldface characters indicate real vectors while matrices will be represented by capitalised letters. Let

$$
\begin{aligned}
& r_{i}=\text { one period (short) rate during }\left[t_{i-1}, t_{i}\right] \\
& f_{i}=\text { cash flow at time } t_{i}, \\
& p_{i}=\text { "running" present value of future cash flow } \\
& \quad \text { at time } t_{i} \text { of the future cash flows } f_{j}, j \geq i \text {. }
\end{aligned}
$$

The short rate $r_{i}$ is assumed to be of the form

$$
r_{i}=g\left(r_{i-1}\right)+v_{i}
$$


where $g(\cdot): \mathbb{R} \mapsto \mathbb{R}$ is a known, deterministic function and the discrete random process $v_{i}, i>0$ satisfies $\mathbb{E}\left(v_{i}\right)=0$ where $\mathbb{E}$ denotes the expectation operator. The initial short rate $r_{0}$ is assumed to be known. When discretised, most standard, single-factor short rate models will reduce to this form. It is also assumed that $v_{i}$ is defined on a finite support such that $\left|r_{i}\right| \leq(1-\epsilon)$ for some $\epsilon>0$. From a practical point of view, this is a reasonable requirement.

The discounted present values $p_{i}$ 's of future cash flows may be defined by a recursive relation

$$
\begin{aligned}
p_{N-1} & =\frac{f_{N}}{1+r_{N}}, \\
p_{i} & =\frac{p_{i+1}+f_{i+1}}{1+r_{i+1}}, i \in[0, N-2] .
\end{aligned}
$$

This relationship may be written in a matrix-vector form as follows.

Fact 1 The future cash flow and its present value at each time $t_{i}$ may be shown to be related by

$$
\mathbf{f}=(Q+R) \mathbf{p}
$$

where

$$
\begin{aligned}
& {[Q]_{i j}=1 \text { if } i=j} \\
& =-1 \text { if } i=j+1 \\
& =0 \text { otherwise. } \\
& {[R]_{i j}=r_{N-i+1} \text { if } i=j \text {, }} \\
& =0 \text { otherwise. } \\
& \mathbf{f}=\left[\begin{array}{llll}
f_{N} & f_{N-1} & \cdots & f_{1}
\end{array}\right]^{T} \\
& \mathbf{p}=\left[\begin{array}{llll}
p_{N-1} & p_{N-2} & \cdots & p_{0}
\end{array}\right]^{T},
\end{aligned}
$$

where $T$ denotes transpose of a matrix or a vector.

Proof : This may easily be proved using (1) .

Given the information concerning the distribution of $r_{i}$ and $f_{i}$, the solution to the system of linear equations (2) provides us with the statistics of the running present value $p_{i}$ at time $t_{i}$. In particular, if the cash flows consist of coupons and principal repayment of a default-free bond, the expected value $\mathbb{E}\left(p_{0}\right)$ represents the bond price at time $t_{0}$.

\section{Approximation of the discounted present value vector}

The next result provides a uniform approximation of the statistics of vector $\mathbf{p}$. 
Theorem 2 Suppose $\max _{i}\left|r_{i}\right|<1, f_{i}$ satisfies the condition $\max _{i}\left|f_{i}\right|<\gamma$ for some $\gamma<\infty$ and the inverse of $(Q+R)$ exists with probability 1 . Also assume that $\mathbb{P}\left(\left\|Q^{-1} R\right\|_{2}<1\right)=1$. Define

$$
\mathbf{p}=(Q+R)^{-1} \mathbf{f}, \mathbf{p}^{M}=\sum_{i=0}^{M}\left(-Q^{-1} R\right)^{i} Q^{-1} \mathbf{f}
$$

with $\left(-Q^{-1} R\right)^{0}=I$. Then

$$
\begin{gathered}
\mathbf{p}^{M} \rightarrow \mathbf{p} \text { with probability } 1 \\
\lim _{M \rightarrow \infty} \mathbb{E}\left(\left\|\mathbf{p}^{M}-\mathbf{p}\right\|_{2}^{2}\right)=0
\end{gathered}
$$

Proof : See the appendix.

Remark 3 The first two assumptions of theorem 2 viz. uniformly bounded cash flows and short rate bounded by $100 \%$ are made to conform with the pragmatic reality of the financial market. The third assumption is justified by the fact that the inverse of $(Q+R)$ exists (a.s.) provided $\min _{i} r_{i}>0$ a.s. We shall return to the issue of interpretation and justification of the last assumption on the size of $Q^{-1} R$ later in this section.

Remark 4 If $r$ is a deterministic non-negative scalar bounded by unity, the expression

$$
\frac{f}{1+r} \approx \sum_{i=0}^{M}(-r)^{i} f
$$

follows trivially from the Taylor's expansion around $r=0$. The multivariate, probabilistic generalisation in theorem 2 (as implied by the definition of $\mathbf{p}^{M}$ ) does not follow easily from the scalar deterministic version, as can be seen from the proof given in the appendix.

The expression for $\mathbf{p}^{M}$ is a multivariate polynomial in $r_{i}$ and $f_{i}$ and is thus significantly simpler than the expression for $\mathbf{p}$ which involves matrix inversion. In particular, the moments of $\mathbf{p}^{M}$ are defined in terms of (joint) moments of $r_{i}$ and $f_{i}$. Thus a uniform approximation to the moments of the present value (in the sense of theorem 1) may be found using the moments of future cash flows and the interest rates.

The computation involved in finding the moments of $\mathbf{p}^{M}$ is simpler than it appears. First, the matrices $Q^{-1}, Q^{-1} R$ and the vector $Q^{-1} \mathbf{f}$ have particularly simple forms:

\section{Fact 5}

$$
\begin{aligned}
{\left[Q^{-1}\right]_{i j} } & =1 \text { if } i \geq j \\
& =0 \text { otherwise } \\
{\left[Q^{-1} R\right]_{i j} } & =r_{N-j+1} \text { if } i \geq j \\
& =0 \text { otherwise, } \\
{\left[Q^{-1} \mathbf{f}\right]_{j} } & =\sum_{i=1}^{j} f_{N-i} .
\end{aligned}
$$


It is instructive to examine what these matrices look like. For $N=3$, we have

$$
\begin{aligned}
Q^{-1} R & =\left[\begin{array}{lll}
r_{3} & 0 & 0 \\
r_{3} & r_{2} & 0 \\
r_{3} & r_{2} & r_{1}
\end{array}\right], \\
Q^{-1} \mathbf{f} & =\left[\begin{array}{c}
f_{3} \\
f_{3}+f_{2} \\
f_{3}+f_{2}+f_{1}
\end{array}\right] .
\end{aligned}
$$

Using fact 5, one may easily establish a closed-form expression for the approximation of $p_{0}$. In particular, the second order approximation for a zero coupon bond (with $f_{i}=0$ for $i=1,2, \ldots, N-1$ and $\left.f_{N}=1\right)$ is stated below.

\section{Fact 6}

$$
\mathbb{E}\left(\mathbf{e}_{\mathbf{1}}^{T} \mathbf{p}^{2}\right)=1-\mathbb{E}\left(\sum_{i=1}^{N} r_{i}\right)+\mathbb{E}\left(\sum_{\substack{i, j=1 \\ i \leq j}}^{N} r_{i} r_{j}\right)
$$

where $\mathbf{e}_{\mathbf{1}}=\left[\begin{array}{llll}0 & 0 & \cdots & 1\end{array}\right]^{T}$ is a unit vector.

Theorem 2 together with fact 5 demonstrates how to build a uniformly convergent approximation to the present price of a general cash flow as defined by

$$
\mathbb{E}\left(\sum_{i=1}^{N} \frac{f_{i}}{\prod_{j=1}^{i}\left(1+r_{j}\right)}\right)
$$

in terms of joint moments $\mathbb{E}\left(\left(\prod_{j=1}^{i} r_{j}\right) f_{i}\right)$. Unless otherwise specified, it is assumed that all the expectations are conditional on the information at time $t_{0}$. An approximation to future prices $p_{i}$ of cash flow based on today's information is also obtained. Further, note that $\mathbb{E}\left(\mathbf{p}^{M}\left(\mathbf{p}^{M}\right)^{T}\right)$ may also be expressed in terms of joint moments of $r_{i}$ and $f_{i}$. Thus the variance (and higher moments) of the present and future values of cash flows may be approximated using this method.

We now return to the assumption on the size of $Q^{-1} R$ in theorem 2 and its implications.

Lemma 7 (i) With probability 1 ,

$$
\left\|\mathbf{p}^{M}-\mathbf{p}\right\| \leq \frac{\left\|Q^{-1} R\right\|^{M+1}}{1-\left\|Q^{-1} R\right\|}\left\|Q^{-1} \mathbf{f}\right\|
$$

holds for any vector-induced norm $\|\cdot\|$, provided $\left\|Q^{-1} R\right\|<1$ with probability 1. 
(ii) If $r_{\text {min }}, r_{\max }$ are positive constants such that, with probability $1, r_{i} \in\left(r_{\min }, r_{\max }\right) \forall i$, then

$$
\left\|Q^{-1} R\right\|_{2} \in\left(r_{\min } \sqrt{\frac{N+1}{2}}, r_{\max } \sqrt{\frac{N(N+1)}{2}}\right)
$$

holds with probability 1.

(1) If $r_{\min }, r_{\max }$ are defined as above, then for any $\epsilon>0, \exists$ a matrix norm $\|\cdot\|$ s.t. $\left\|Q^{-1} R\right\| \in$ $\left(r_{\min }, r_{\max }+\epsilon\right)$.

Proof : See the appendix.

Note that $r_{\max }$ is a bound on interest rate per period which is not annualised and is likely to be a small number. Since $\frac{N(N+1)}{2} \leq N^{2}$, a sufficient condition for the bound $\left\|Q^{-1} R\right\|_{2}<1$ to be satisfied is $N r_{\max }<1$. However, the error bound in (6) is still conservative. As may be seen from its proof, this conservatism stems from the use of trace of a positive semi-definite matrix to bound from above its maximum eigenvalue. Whilst this bound is an equality in the worst case, it is very conservative for well-conditioned matrices. It is hard to impose a constraint on the condition number of $Q^{-1} R$ in terms of a relevant constraint on $r_{i}$. However, the errors in practice seem to be far less than those suggested by (5), as demonstrated in a latter section on pricing of default-free bonds.

\section{Approximation using short rate models for stochastic interest rate}

Next, we address the problem of obtaining moments $\mathbb{E}\left(\prod_{j=1}^{i} r_{j}\right)$. Given a parametrised model of the short rate $r_{k}$ (see James and Webber (2000), chapter 7 for a comprehensive overview of short rate models), it is easy to obtain the joint moments for $i \leq 2$. The moments for $i=3$ are only slightly more involved, but are omitted here for brevity. We consider two different models:

CIR model (Cox, Ingersoll and Ross (1985)): $r_{k+1}=a b+(1-a) r_{k}+\sigma \sqrt{r_{k}} w_{k+1}$

Hull-White Model (Hull and White (1990)): $r_{k+1}=a b+(1-a) r_{k}+\sigma w_{k+1}$

In both cases, $w_{k}$ are zero mean, unit variance, bounded and independent random variables. $a, b$ and $\sigma$ are either constants or are known functions of $k$. Furthermore, it is assumed that

$$
\mathbb{P}\left(r_{i} \in[0,1)\right)=1
$$

Euler-discretisation of classical Hull-White and Cox-Ingersoll-Ross models will yield models which look similar to the ones above; however, it is worth remembering the crucial constraint above implies a non-Gaussian noise on a finite support. Condition (9) is crucial for the approximation technique to converge, i.e. for theorem 2 to hold. Of course, the one-period interest rate being bounded by unity is a perfectly reasonable assumption from a practical 
point of view. Note that the time period for discretisation is assumed to be unity. This causes no loss of generality; for a period $\Delta$, one would get the same results provided one replaces $a$ with $a \Delta$ and $\sigma$ with $\sigma \sqrt{\Delta}$.

For these models, one may obtain expressions for the first two conditional moments as follows.

Lemma 8 (1) If (7) holds, then

$$
\begin{aligned}
\mathbb{E}\left(r_{i}\right) & =(1-a)^{i} r_{0}+a b \sum_{l=0}^{i-1}(1-a)^{l} \text { and } \\
\mathbb{E}\left(r_{i} r_{j}\right) & =\mathbb{E}\left(r_{i}\right) \mathbb{E}\left(r_{j}\right)+\sigma^{2} \sum_{l=1}^{j}(1-a)^{i+j-2 l} \mathbb{E}\left(r_{l-1}\right) .
\end{aligned}
$$

(2) If (8) holds, then

$$
\begin{aligned}
\mathbb{E}\left(r_{i}\right) & =(1-a)^{i} r_{0}+a b \sum_{l=0}^{i-1}(1-a)^{l} \text { and } \\
\mathbb{E}\left(r_{i} r_{j}\right) & =\mathbb{E}\left(r_{i}\right) \mathbb{E}\left(r_{j}\right)+\sigma^{2} \sum_{l=1}^{j}(1-a)^{i+j-2 l} .
\end{aligned}
$$

In both cases, $i \geq j$ is assumed without loss of generality.

Proof : This may be proved by straightforward algebraic manipulation of (7) and (8).

The above result, along with theorem 2 and fact 5, may be used to derive a second order approximation of the present value of any cash flow under a given short rate dynamics.

\section{Applications}

The linear algebraic approach described so far is quite generic and can be used in the approximate pricing of any conceivably uncertain cash flows under stochastic interest rates. In essence, our proposed technique may be seen as an approach which can map the moments of $\left(\Pi_{j} r_{j}\right) f_{i}$ into the moments of the present value vector, without resorting to the use of full distribution information. If the expected value and perhaps the variance of the present value vector (and not its full set of outcomes) are mainly of interest, using the method developed here may be computationally more efficient than using distribution-based methods, such as lattice-based or Monte Carlo techniques.

We demonstrate the method by pricing default-free zero coupon bonds. The illustration is motivated by the availability of closed-formulae for the bond price under the short rate models mentioned in the previous section. This allows us to benchmark our approximation with the "true" price of the cash flow. The pricing of defaultable bonds is also briefly elaborated. This exemplifies the pricing methodology where the cash flows $f_{i}$ are also uncertain. 
Pricing Default-free bonds: Numerical Experiments

The method described above is demonstrated here by pricing zero-coupon UK government bonds. Data for UK treasury strips for 8 maturity dates was taken from Datastream on $15^{\text {th }}$ February 2005. The parameters of a CIR model are estimated using simple least-squares (under risk-neutral measure). The parameters obtained are

$$
a=0.7366, b=0.0037, \sigma=0.0049, r_{0}=0.0041 .
$$

The purpose of using cross-sectional data is simply comparing our method to CIR closedform formulae for a realistic set of parameters. Even though the parameters are the same, the classical CIR model and the proxy model which we use are fundamentally different since our proxy is assumed to be defined on a compact support. However, we are interested only in good approximation of the moments of the bond value (with the first moment in the risk-neutral measure being the bond price) and using the proxy model specified in (7) along with lemma 8 seems to be adequate for the job.

For these parameters, the prices of 1 Euro payable after $\tau$ periods (months) are compared against $2^{\text {nd }}$ and $3^{\text {rd }}$ order approximation. If the short rate process follows CIR dynamics, the exact price $P_{\tau}$ of 1 Euro payable after time $\tau$ is given by

$$
\begin{aligned}
P_{\tau} & =A_{\tau} e^{-B_{\tau} r} \quad \text { where } \\
A_{\tau} & =\left(\frac{2 \gamma e^{(a+\gamma) \tau / 2}}{(\gamma+a)\left(e^{\gamma \tau}-1\right)+2 \gamma}\right)^{\frac{2 a b}{\sigma^{2}}} \\
B_{\tau} & =\frac{2\left(e^{\gamma \tau}-1\right)}{(\gamma+a)\left(e^{\gamma \tau}-1\right)+2 \gamma} \\
\gamma & =\sqrt{a^{2}+2 \sigma^{2}}
\end{aligned}
$$

and $r$ is the current rate of interest per period. The results are shown in table 1.

\section{Table 1 approximately here}

It can be seen that the percentage error in all cases is below $0.05 \%$ (respectively, below $1.1 \%$ ) for third order (respectively, second order) approximation.

\section{Defaultable Bonds}

A simple default structure is assumed. An exogenous and non-negative random variable $\tau$ represents the time of default. In general, $\tau$ will be correlated to $r_{i}$. A deterministic recovery function $\alpha_{i}$ is assumed such that if the default occurs in $\left[t_{i-1}, t_{i}\right)$, the recovery value is $\alpha_{i}$. The nominal value of coupon payment at time $t_{i}$ is denoted as $f_{i}^{0}$. It is easy to see that the 
price of defaultable bond at time $t_{0}$ is given by

$$
\mathbb{E}\left(p_{0}\right)=\sum_{i=1}^{N} \mathbb{E}\left(\frac{f_{i}}{\prod_{j=1}^{i}\left(1+r_{j}\right)}\right) .
$$

In the special case when $\tau$ and $r_{i}$ are uncorrelated, we have

$$
\mathbb{E}\left(f_{i}\right)=\mathbb{P}\left(\tau \geq t_{i}\right) f_{i}^{0}+\mathbb{P}\left(\tau \in\left[t_{i-1}, t_{i}\right)\right) \alpha_{i}
$$

and an approximation to bond price may be constructed as

$$
\left[\mathbf{p}^{M}\right]_{0}=\sum_{i=0}^{M} \mathbb{E}\left\{e_{1}^{T}\left(Q^{-1} R\right)^{i}\right\} \mathbb{E}\left(Q^{-1} \mathbf{f}\right) .
$$

with matrices $Q$ and $R$ and unit vector $e_{1}$ defined as in the second section. Given a distribution of $\tau$ (as in the structural approach of Black and Cox (1976) amongst others), this enables us to approximate the bond price. As mentioned in the third section, it is also possible to find the variance of the value of bond by finding $\mathbb{E}\left(\mathbf{p}^{M}\left(\mathbf{p}^{M}\right)^{T}\right)$.

\section{Conclusion}

The contributions of this work are three-fold. First, it introduces a new linear algebraic set-up for valuing cash flows. Secondly, new expressions are derived for a convergent approximation to the random vector representing the cash flow and for analysing the error in this approximation. This analysis covers a large variety of cash flow pricing problems under interest rate uncertainty. Finally, it is shown that the performance of low order approximation for pricing zero coupon bonds is comparable with the closed-form expressions obtained by the CIR model under the relevant short rate dynamics.

Application of this method to value a future cash flows arising in investment appraisal and project development problems is a topic of current research. Another topic of interest is the pricing of perpetuities. The conditions on existence of stable distributions as $N$ goes to infinity were handled in the past by Cairns (1995) and Dufresne (1990). It would be theoretically interesting and practically relevant to investigate whether the approximations similar to the ones suggested in this paper may be derived for the limiting distributions when perpetuities are involved.

\section{Acknowledgements}

The first author gratefully acknowledges helpful comments from Dr Manti Mendi at Brunel University. 


\section{Appendix}

\section{Proof of theorem 1}

The proof rests on the following standard results in probability (see, e.g., Grimmett and Stirzaker (2001), theorem 7.4):

Lemma 9 (1) If $\sum_{N} \mathbb{P}\left(\left|\mathbf{X}_{N}-\tilde{\mathbf{X}}\right|>\epsilon\right)<\infty$ for all $\epsilon>0$, then $\mathbf{X}_{N} \rightarrow \tilde{\mathbf{X}}$ with probability 1.

(2) If $\mathbf{X}_{N} \rightarrow \tilde{X}$ in probability and $\mathbb{P}\left(\left\|\mathbf{X}_{N}\right\|_{2}<k\right)=1 \forall N$ for some $k$, then

$$
\lim _{N \rightarrow \infty} \mathbb{E}\left(\left|\mathbf{X}_{N}-\tilde{\mathbf{X}}\right|^{2}\right)=0
$$

From proof of theorem 7.4 in Grimmett and Stirzaker (2001), it is easy to see that analogous result holds for vector-valued random variables bounded elementwise from above and below, using the bound $\max _{i}(|\mathbf{x}|)_{i} \leq\|\mathbf{x}\|_{2}$ for the two-norm. The proof of the first part of theorem 1 is based on deriving an upper bound for $\mathbb{P}\left(\left\|\mathbf{p}^{M}-\mathbf{p}\right\|>\epsilon\right)$ and then showing that the summation of these upper bounds over $M$ is finite.

First, a simple result is needed to derive the necessary bound:

Lemma 10 Let $Q, R$ be as defined in fact 1 and let $\rho$ denote the spectral radius. Then

$$
\max _{i}\left|r_{i}\right|<1 \quad \Rightarrow \rho\left(Q^{-1} R\right)<1
$$

Proof : Eigenvalues of any lower triangular matrix are given by its diagonal entries, which in the case of $Q^{-1} R$ are $r_{i}$ 's.

Since $\mathbb{P}\left(\rho\left(Q^{-1} R\right)<1\right)=1$, the following power series expansion converges with probability 1 (see, e.g. lemma 5.6.10 and corollary 5.6.16 in Horn and Johnson (1999)):

$$
\begin{aligned}
(Q+R)^{-1} & =\left(I-Q^{-1} R\right)^{-1} Q^{-1} \\
& \left.=\sum_{i=0}^{\infty}\left(-Q^{-1} R\right)^{i}\right) Q^{-1}
\end{aligned}
$$

Then

$$
\mathbb{P}\left(\mathbf{p}=\sum_{i=0}^{\infty}\left(-Q^{-1} R\right)^{i} Q^{-1} \mathbf{f}\right)=1
$$


Suppose that $\mathbb{P}\left(\left\|Q^{-1} R\right\|_{2} \leq \alpha\right)=1$ for some $\alpha<1$. Also, let $\left\|Q^{-1} \mathbf{f}\right\|_{2}=\beta$. For a given $\epsilon>0$, let $M_{\epsilon}$ be the smallest integer such that $\frac{\alpha^{M_{\epsilon}+1} \beta}{1-\alpha} \leq \epsilon$. Now, for any $M_{0}>M_{\epsilon}$,

$$
\begin{aligned}
& \mathbb{P}\left(\left\|\mathbf{p}^{M_{0}-1}-\mathbf{p}\right\|_{2}>\epsilon\right)=\mathbb{P}\left(\left\|\left\{\sum_{i=M_{0}}^{\infty}\left(-Q^{-1} R\right)^{i}\right\} Q^{-1} \mathbf{f}\right\|_{2}>\epsilon\right) \\
& =0, \quad \text { since, with probability } 1 \\
& \left\|\left\{\sum_{i=M_{0}}^{\infty}\left(-Q^{-1} R\right)^{i}\right\} Q^{-1} \mathbf{f}\right\|_{2} \leq \frac{\alpha^{M_{0}+1} \beta}{1-\alpha} \leq \frac{\alpha^{M_{\epsilon}+1} \beta}{1-\alpha} \leq \epsilon .
\end{aligned}
$$

Next, for any $\epsilon>0$,

$$
\sum_{M=0}^{\infty} \mathbb{P}\left(\left\|\mathbf{p}^{M}-\mathbf{p}\right\|_{2}>\epsilon\right) \leq \sum_{M=0}^{M_{\epsilon}} \mathbb{P}\left(\left\|\mathbf{p}^{M}-\mathbf{p}\right\|_{2}>\epsilon\right) .
$$

The proof of part (1) is completed by noting that $M_{\epsilon}$ is finite for any $\epsilon>0$ and using the first part of lemma 9. Part (2) may be proven by noting that convergence with probability 1 implies convergence in probability and that

$$
\mathbb{P}\left(\left\|\mathbf{p}^{M}\right\|_{2} \leq \frac{\beta}{1-\alpha}\right)=1
$$

holds for all non-negative integers $M$.

\section{Proof of lemma 7}

For any induced norm $\|\cdot\|$ such that $\left\|Q^{-1} R\right\|<1$ with probability 1 , we have

$$
\begin{aligned}
\left\|\mathbf{p}^{M}-\mathbf{p}\right\|_{2} & =\left\|\sum_{i=M+1}^{\infty}\left(Q^{-1} R\right)^{i} Q^{-1} \mathbf{f}\right\| \\
& \leq \sum_{i=M+1}^{\infty}\left\|\left(Q^{-1} R\right)^{i} Q^{-1} \mathbf{f}\right\| \\
& \leq \sum_{i=M+1}^{\infty}\left\|Q^{-1} R\right\|^{i}\left\|Q^{-1} \mathbf{f}\right\|
\end{aligned}
$$

from which result (5) follows. To prove the upper bound in (6), the definition of 2-norm implies that

$$
\begin{aligned}
\left\|Q^{-1} R\right\|_{2} & =\max _{i} \sigma_{i}\left(Q^{-1} R\right)=\sqrt{\max _{i} \lambda_{i}\left(Q^{-1} R R^{T}\left(Q^{-1}\right)^{T}\right)} \\
& \leq \sqrt{\operatorname{trace}\left(Q^{-1} R R^{T}\left(Q^{-1}\right)^{T}\right)} \leq \sqrt{\sum_{i=1}^{N} i r_{\max }^{2}}
\end{aligned}
$$

where $\lambda_{i}(A)$ and $\sigma_{i}(A)$ are the $i^{\text {th }}$ largest eigenvalue and $i^{\text {th }}$ largest singular value of matrix $A$, respectively. The first inequality is immediate since the maximum eigenvalue of a matrix 
is bounded by its trace (e.g. Horn and Johnson (1999), chapter 1); and the second inequality may easily be proved from the structure of $Q^{-1} R$ in fact 5. Similarly, the lower bound in (6) follows from the inequalities

$$
\left\|Q^{-1} R\right\|_{2} \geq \sqrt{\frac{\operatorname{trace}\left(Q^{-1} R R^{T}\left(Q^{-1}\right)^{T}\right)}{N}} \geq \sqrt{\frac{\sum_{i=1}^{N} i r_{\min }^{2}}{N}} .
$$

Finally, note that

$$
r_{i} \in\left(r_{\min }, r_{\max }\right) \forall i \Rightarrow \rho\left(Q^{-1} R\right) \in\left(r_{\min }, r_{\max }\right)
$$

(also see proof of lemma 10). The last result in lemma 7 then follows directly by employing lemma 5.6.10 in Horn and Johnson (1999): given any $\epsilon>0$, there is a matrix norm such that

$$
\rho\left(Q^{-1} R\right) \leq\left\|Q^{-1} R\right\| \leq \rho\left(Q^{-1} R\right)+\epsilon .
$$

\section{References}

Bielecki, T. and M. Rutkowski. 2004. Credit Risk: Modeling, Valuation and Hedging. Springer-Verlag.

Black, F., E. Derman and W. Toy. 1990. "A one-factor model of interest rates and its applications to treasury bond options." Financial Analysts Journal pp. 33-39.

Black, F. and J. Cox. 1976. "Valuing Corporate Securities: Some Effects of Bond Indenture Provisions." Journal of Finance 31:351-367.

Bowers, N., H. Gerber, J. Hickman, D. Jones and C. Nesbitt. 1997. Actuarial Mathematics. Schaumburg, Illinois: Society of Actuaries.

Brennan, M. and E. Schwartz. 1979. "A continuous time approach to the pricing of bonds." Journal of Banking and Finance 3:133-155.

Burnecki, K., A. Marciniuk and A. Weron. 2003. "Annuities under Random Rates of Interest - Revisited." Insurance: Mathematics and Economics 32:457-460.

Cairns, A.J.G. 1995. "The present Value of a series of Cashflows: Convergence in a Random Environment." Astin Bulletin 25:81-94.

Cheyette, O. 1998. Term structure dynamics and mortgage valuation, in Monte Carlo: Methodologies and Applications for Pricing and Risk Management. London, UK: edited by B. Dupire, Risk Books pp. 191-204.

Cox, J., J. Ingersoll and S. Ross. 1985. "A theory of the term structure of interest rates." Econometrica 53:385-407.

Dufresne, D. 1990. "The distribution of a perpetuity, with applications to risk theory and pension funding." Scandinavian Actuarial Journal 1-2:39-79.

Grimmett, G. and D. Stirzaker. 2001. Probability and Random Processes. Oxford University Press.

Hamilton, J. 1988. "Rational-expectations econometric analysis of changes in regime: An investigation of the term structure of interest rates." Journal of Economic Dynamics and Control 12:385-423. 
Ho, T. and S. Lee. 1986. "Term structure movements and the pricing of interest rate contingent claims." Journal of Finance 56:1011-1029.

Horn, R.A. and C. R. Johnson. 1999. Matrix Analysis. Cambridge University Press.

Hull, J. and A. White. 1990. "Pricing Interest Rate Derivative Securities." Review of Financial Studies 3:573-592.

James, J. and N. Webber. 2000. Interest Rate Modelling. John Wiley and Sons.

Jarrow, R. 1996. Modelling Fixed Income Securities and Interest Rate Options. New York: Mcgraw Hill.

Jarrow, R.A. 2002. Modelling Fixed Income Securities and Interest Rate Options. Stanford University Press.

J.Feinberg. N.d. "On the Universality of the probability distribution of the product $B^{-1} X$ of random matrices." http://arxiv.org/abs/math/PR0204312.

Kellison, S. 1991. The Theory of Interest. Irwin.

Koch, I. and A. De Schepper. 2004. "General annuities under truncate stochastic interest rates." presented at the 7th International Congress on Insurance: Mathematics and Economics, ISFA, University of Lyon, France.

Schepper, A. De, A. Goovaerts, M. Kaas and D. Vyncke. 1997. "A recursive scheme for perpetuities with random positive interest rates." Scandinavian Actuarial Journal 1:1-10.

Vasicek, O. 1977. "An equilibrium characterisation of the term structure." Journal of Financial Economics 5:177-188.

Zaks, A. 2001. "Annuities under random rates of interest." Insurance: Mathematics and Economics 28:1-11. 
Table 1

\begin{tabular}{|c|c|c|c|}
\hline$\tau$ & C.I.R. price & {$\left[\mathbf{p}^{2}\right]_{0}$} & {$\left[\mathbf{p}^{3}\right]_{0}$} \\
\hline 6 & 0.97754 & 0.97758 & 0.97758 \\
12 & 0.95606 & 0.95616 & 0.95614 \\
24 & 0.91450 & 0.91478 & 0.91465 \\
48 & 0.83673 & 0.83797 & 0.83696 \\
96 & 0.70047 & 0.70803 & 0.70027 \\
\hline
\end{tabular}

